\title{
A study of head and neck cancer treatment and survival among indigenous and non-indigenous people in Queensland, Australia, 1998 to 2004
}

\author{
Suzanne P Moore ${ }^{1,3^{*}}$, Adèle C Green ${ }^{1,5}$, Gail Garvey ${ }^{1}$, Michael D Coory ${ }^{4}$ and Patricia C Valery ${ }^{1,2^{*}}$
}

\begin{abstract}
Background: Overall, Indigenous Australians with cancer are diagnosed with more advanced disease, receive less cancer treatment and have poorer cancer survival than non-Indigenous Australians. The prognosis for Indigenous people with specific cancers varies however, and their prognosis for cancers of the head and neck is largely unknown. We therefore have compared clinical characteristics, treatment and survival between Indigenous and non-Indigenous people diagnosed with head and neck cancer in Queensland, Australia.
\end{abstract}

Methods: Rates were based on a cohort of Indigenous people $(n=67)$, treated in public hospitals between 1998 and 2004 and frequency-matched on age and location to non-Indigenous cases $(n=62)$ also treated in the public health system. Data were obtained from hospital records and the National Death Index. We used Pearson's Chisquared analysis to compare categorical data (proportions) and Cox proportional hazard models to assess survival differences.

Results: There were no significant differences in socioeconomic status, stage at diagnosis or number and severity of comorbidities between Indigenous and non-Indigenous patients, although Indigenous patients were more likely to have diabetes. Indigenous people were significantly less likely to receive any cancer treatment $75 \% \mathrm{vs}$. 95\%, P = $0.005)$ and, when cancer stage, socioeconomic status, comorbidities and cancer treatment were taken into account, they experienced greater risk of death from head and neck cancer (HR 1.88, 1.10, 3.22) and from all other causes (HR 5.83, 95\% Cl 1.09, 31.04).

Conclusion: These findings show for the first time that Indigenous Australians with head and neck cancer receive less cancer treatment and suggest survival disparity could be reduced if treatment uptake was improved. There is a need for a greater understanding of the reasons for such treatment and survival disparities, including the impact of the poorer overall health on cancer outcomes for Indigenous Australians.

\section{Background}

Overall, Indigenous Australians with cancer are diagnosed with more advanced disease, receive less cancer treatment and have poorer cancer survival than nonIndigenous Australians [1,2]. However, the prognosis for Indigenous people with specific cancers such as those of the head and neck is largely unknown.

The term "cancers of the head and neck" refers to a group of cancers that occur on the lip, tongue, gum,

\footnotetext{
*Correspondence: Suzanne.moore@qimr.edu.au; Patricia.Valery@menzies.edu. au

'Cancer and Population Studies, Queensland Institute of Medical Research, Herston Rd, Brisbane, 4060, Australia

Full list of author information is available at the end of the article
}

oral cavity, the sinuses, nose, salivary glands and throat [3]. Most head and neck tumours are squamous cell carcinomas (SCC) for which significant risk factors include smoking and alcohol exposure, and increasingly, infection by human papillomavirus (HPV), which now reportedly accounts for $25 \%$ of cases in the United States [4]. Head and neck cancers rank as the fifth most commonly occurring cancer type for Australia overall [5] and also for Indigenous people in Queensland [6].

Little is known about the incidence and mortality of head and neck cancer among Indigenous relative to non-Indigenous populations in the developed world. Overall incidence of oral and pharyngeal cancers amongst American Indians/Alaskan Natives (AI/AN)

\section{Biomed Central}


was reportedly lower than for whites but varied widely across districts of the United States, and stage distribution was found to be less favourable for AI/AN [7]. In the Indigenous population of the Northern Territory of Australia, the incidence of head and neck cancer is around 32.78 per 100,000 compared to 19.5 per 100,000 for the rest of Australia [8] and higher incidence and mortality from cancer of the oropharynx have previously been reported in the Northern Territory Indigenous population when compared to non-Indigenous people [2]. Whilst incidence was reportedly similar among Indigenous and non-Indigenous people with head and neck cancers in Queensland between 1997 and 2006, mortality was more than three times greater for Indigenous people [6].

It is not known to what extent established prognostic factors such as late stage at diagnosis, the presence and severity of comorbidities or differences in cancer treatment account for the excess mortality among Indigenous Australians with head and neck cancer. Therefore we conducted this population-based study to compare these factors and survival between Indigenous and nonIndigenous Australians diagnosed with this disease.

\section{Methods}

\section{Subjects}

The study was conducted in Queensland, the northeastern state of Australia, which has a population of 4.5 million, 3.6\% of whom identify as Aboriginal, Torres Strait Islander, or both (respectfully referred to here as Indigenous) [9]. All Indigenous people (over 18 years) residing in Queensland and diagnosed with head and neck cancer between 1998 and 2004 were identified through the population-based Queensland Cancer Registry (QCR). To improve the efficiency of this cohort study, Indigenous patients were matched to a random sample of 74 non-Indigenous people with incident head and neck cancer also identified through the Cancer Registry, of corresponding age (within $+/-5 \mathrm{yrs}$ ), sex and location of residence (in terms of degree of remoteness from a major centre).

Clinical data were abstracted from the records at 14 public hospitals to a standard form by one of the researchers (SPM), an experienced nurse, and a former Registrar of the Queensland Cancer Registry. Where records were insufficient, further data were extracted from secondary public hospitals' records. Records were reviewed for diagnostic details such as date, histology and method of diagnosis, cancer stage, treatment and presence of comorbidities. Cancer staging was documented by treating doctors as either Tumour, Nodes and Metastasis (TNM) scores, American Joint Committee on Cancer Staging Scores (I - IV) [10] or as localised cancer, regional spread or metastatic disease; for comparability, TNM and AJCC scores were converted to localised/regional/distant spread using commonly accepted cut- points.

Treatment type, including surgery, radiotherapy and chemotherapy were recorded, as was treatment intention (any intent, curative intent or intention not known). Date, duration and quantity of treatment were also collected. The presence or absence of comorbid conditions was recorded, and a modified Charlson Comorbidity Index Score (referred to here as 'comorbidity score') was assigned (based on severity and number of comorbid conditions and were grouped thus: No score, 1, 2-5 and $5+)$. Specifically, diabetes, cardio and cerebrovascular disease, hypertension, respiratory illness, renal disease, history of previous cancer and other serious comorbidities that were accepted in the Charslon Comorbidity Scoring framework, were assessed [11]. A Socioeconomic score was assigned using the Socio-Economic Index For Areas (SEIFA) index (based on geographical areas of residence ranked into quintiles: 1 representing the most disadvantaged, and 5 the most advantaged) [12]. Date and cause of death were obtained from the Australian National Death Index.

\section{Statistical methods}

Pearson's Chi-squared analysis or Fisher's Exact test were used for categorical data (proportions), t-test for normallydistributed data (means), and non-parametric tests (Kruskal-Wallis Test) for non-normally-distributed data (medians). Crude and adjusted survival analyses were conducted using Cox proportional hazard models. Hazard ratios were adjusted for stage of cancer at diagnosis, presence and severity of comorbidities, socioeconomic status and 'any' or 'curative' cancer treatment and treatment mode, defined as any curative surgery, radiotherapy or chemotherapy or combination of these. The reference category was "non-Indigenous" unless otherwise stated. All analyses were calculated using Statistics Package for the Social Sciences (PASW) for Windows version 17.0.

Ethical approval to conduct the study was obtained from the Queensland Health Department, all hospitals where data collection took place, and the Queensland Institute of Medical Research. An Indigenous Reference Group was established to inform the study investigators about cultural matters and the translation of results to the community.

\section{Results}

\section{Demographics and Clinical features}

As all Indigenous patients had attended a Queensland public hospital, two non-Indigenous patients who were primarily treated in a private facility were excluded. In addition, cases with cancer of the lip, which mostly arises on the sun-exposed non-mucosal epithelium in white-skinned people [13], were excluded as 7 of the 8 
cancers of the lip in the study occurred in the non-Indigenous cohort. Medical records were not available for two Indigenous and three non-Indigenous patients, leaving 67 Indigenous and 62 non-Indigenous people with head and neck cancers for study.

Mean age was 56 years for Indigenous and 55 years for non-Indigenous people and there was no difference in the proportion of males and females, degree of remoteness from a major centre or socioeconomic index between the two groups (Table 1). The percentages of Indigenous and non-Indigenous patients with squamous cell carcinoma were similar (Indigenous 96\% vs. nonIndigenous 90\%). There was no significant difference in stage at diagnosis (Table 1): the majority of people were diagnosed with regional spread of their cancer (Indigenous $57 \%$, non-Indigenous $65 \%$ ) and no Indigenous or non-Indigenous people from urban regions were diagnosed with a late stage cancer. There was little difference in the comorbidity score between Indigenous and nonIndigenous people (Table 1) and although a greater proportion of Indigenous people had diabetes ( $12 \%$ vs. $3 \%)$, this difference was not statistically significant $(\mathrm{P}=0.09)$.

Indigenous people with cancer of the head and neck were significantly less likely to receive any cancer treatment (either palliative treatment, treatment with curative intent or treatment with intention unknown) than their non-Indigenous counterparts $(75 \%$ vs. $95 \%, \mathrm{P}=0.005)$. Furthermore, when people with metastatic cancer or uncertain stage were excluded, Indigenous patients were less likely to receive treatment with curative intent $(48 \%$ vs. $76 \%, \mathrm{P}=0.002)$ (Table 2). Compared to non-Indigenous patients, the pattern of curative treatment received by Indigenous patients was significantly different $(P=0.03)$. The most common modes of treatment for Indigenous patients were surgery only (36\%) or surgery and radiotherapy (25\%) compared to surgery and radiotherapy (40\%) and chemotherapy (30\%) for their non-Indigenous counterparts (Table 2).

The characteristics of those who did not receive treatment were examined more closely. Indigenous people aged over 60 were less likely to receive any treatment than non-Indigenous people of similar age (40\% did not receive treatment compared to $5 \%$ ), as were Indigenous men when compared to non-Indigenous men (30\% vs. $5 \%)$. Indigenous people with regional spread of disease ( $27 \%$ vs. $5 \%$ ), those from urban, rural and remote areas and those from all socioeconomic strata, were also less likely to receive any treatment than their non-Indigenous counterparts. The comorbidity score was similar for Indigenous people and non-Indigenous people who did not receive treatment. When only cases with non-metastatic disease were compared, older Indigenous people ( $42 \%$ vs. $31 \%$ ), those from the most accessible areas (26\% vs. $8 \%)$, those from the most remote areas $(32 \%$ vs. $15 \%)$, and the most socially disadvantaged ( $55 \%$ vs. $31 \%$ ) were less likely to receive treatment with curative intent than their non-Indigenous counterparts. $46 \%$ of Indigenous people with a comorbidity score of zero did not receive treatment compared to $68 \%$ of non-Indigenous people with the same score but the numbers were too small to show significance (data not shown).

The pattern of curative treatment received by Indigenous patients without metastatic disease was significantly different from that of non-Indigenous patients (Table 2). However there were no significant differences in time to surgery or radiotherapy for those who received cancer treatment and Indigenous people were as likely to complete radiotherapy and received a similar course of radiotherapy as their non-Indigenous counterparts.

\section{Survival}

There was no statistically significant difference between the groups regarding recurrence recorded in the medical chart (Table 2). The pattern of death was significantly different when Indigenous patients were compared to non-Indigenous patient; $79 \%$ of Indigenous patients were deceased by the end of the follow up period compared to $52 \%$ of non-Indigenous patients (Table 2) with risk of all cause-specific death doubled for Indigenous people (HR 2.19 95\% CI 1.36, 3.53) (Table 3). When time to cancer death was adjusted for cancer stage, the hazard ratio was 2.45 (95\% CI 1.51, 3.96), with little variation when further adjusted for socioeconomic status and comorbidities. When further adjusted for overall treatment, the difference was less but remained statistically significant (HR $1.88 \%$ CI 1.10, 3.22). However, when curative treatment and mode of treatment were taken into account, the survival difference was no longer evident (Table 3 ).

Indigenous patients were also more likely to die of causes other than cancer when compared to non-Indigenous patients $(\mathrm{n}=9(17 \%)$ vs. $\mathrm{n}=3,(9 \%))$, though the difference was not statistically significant (Table 2). When we compared time to death from other causes between the two groups (Table 3), we found head and neck cancer Indigenous patients to be 7 times more likely than non-Indigenous patients to die of causes other than the cancer after diagnosis (HR, unadjusted, 7.33 95\% CI $1.52,35.18, \mathrm{P}=0.01$ ) and this was largely unaffected when adjusted separately for cancer stage, presence and severity of comorbidities and diabetes (data not shown), or after adjustment for any cancer treatment (fully adjusted HR 5.83 95\% CI 1.09, 31.04, P = 0.039).

\section{Discussion}

This study reports clinical characteristics, treatment and survival for 67 Indigenous people diagnosed with head and neck cancer between 1998 and 2004 in Queensland, Australia. As the number of cases was small, and the data collection restricted to information available from 
Table 1 Demographic and clinical details, for Indigenous and non-Indigenous patients with cancer of the head and neck in Queensland Australia, 1998- 2004

\begin{tabular}{|c|c|c|c|}
\hline & \multicolumn{2}{|c|}{ Indigenous Status } & \multirow[t]{2}{*}{$P$ value } \\
\hline & $\begin{array}{c}\text { Indigenous } \\
N=67 \\
N \%\end{array}$ & $\begin{array}{c}\text { Non-Indigenous } \\
N=62 \\
N \%\end{array}$ & \\
\hline \multicolumn{4}{|l|}{ Cancer site (ICD code) } \\
\hline Floor of Mouth (CO4) & $5(8)$ & $7(11)$ & \\
\hline Gum (CO3) & $1(2)$ & $3(5)$ & \\
\hline Hypopharynx (C13) & $1(2)$ & $2(3)$ & \\
\hline Lip, oral cavity, pharynx - ill defined (C14) & $9(13)$ & $6(10)$ & \\
\hline Nasopharynx (C11) & $3(5)$ & $2(3)$ & \\
\hline Oropharynx (C10) & $5(8)$ & $2(3)$ & 0.26 \\
\hline Palate (C05) & $8(12)$ & $2(3)$ & \\
\hline Parotid (C07) & $3(5)$ & $7(11)$ & \\
\hline Pyriform Sinus (C12) & $7(10)$ & $7(11)$ & \\
\hline Tongue (C01- C02) & $15(22)$ & $20(32)$ & \\
\hline Tonsil ( C09) & $5(8)$ & $4(7)$ & \\
\hline Unspecified Salivary glands (C08) & $1(2)$ & 0 & \\
\hline Unspecified mouth (C06) & $4(6)$ & 0 & \\
\hline \multicolumn{4}{|l|}{ Age } \\
\hline 18- 39 & $2(3)$ & $4(7)$ & \\
\hline $40-59$ & $40(60)$ & $39(63)$ & 0.52 \\
\hline $60+$ & $25(37)$ & $19(30)$ & \\
\hline \multicolumn{4}{|l|}{ Sex } \\
\hline Male & $47(70)$ & $43(70)$ & 0.92 \\
\hline Female & $20(30)$ & $19(30)$ & \\
\hline \multicolumn{4}{|l|}{ Area of remoteness (ARIA) } \\
\hline Highly accessible & $11(17)$ & $7(11)$ & \\
\hline Accessible & $9(14)$ & $10(16)$ & 0.28 \\
\hline Moderately accessible & $25(37)$ & $33(53)$ & \\
\hline Remote & $9(13)$ & $6(10)$ & \\
\hline Very remote & $13(19)$ & $6(10)$ & \\
\hline \multicolumn{4}{|l|}{ Socioeconomic status (SEIFA) } \\
\hline 1 Most disadvantaged & $26(39)$ & $13(21)$ & \\
\hline 2 Low to Intermediate advantage & $13(19)$ & $20(32)$ & \\
\hline 3 Intermediate advantage & $13(19)$ & $13(21)$ & 0.19 \\
\hline 4 Advantaged & $11(16)$ & $12(19)$ & \\
\hline 5 Most advantaged & $4(6)$ & $2(3)$ & \\
\hline 9 Not known & 0 & $2(3)$ & \\
\hline \multicolumn{4}{|l|}{ Stage at diagnosis (SEER score) } \\
\hline Localised cancer & $23(34)$ & $15(24)$ & \\
\hline Regional Spread & $38(57)$ & $40(65)$ & 0.41 \\
\hline Distant metastasis & $5(7)$ & $7(11)$ & \\
\hline Not sure & $1(2)$ & 0 & \\
\hline \multicolumn{4}{|c|}{ Charlson Comorbidity Index (Scores grouped) } \\
\hline 0 & $42(63)$ & $42(68)$ & \\
\hline 1 & $16(24)$ & $8(13)$ & 0.30 \\
\hline $2-5$ & $9(13)$ & $11(18)$ & \\
\hline $5+$ & 0 & $1(2)$ & \\
\hline \multicolumn{4}{|l|}{ Specific comorbidities } \\
\hline \multicolumn{4}{|l|}{ Diabetes } \\
\hline No & $59(88)$ & $60(97)$ & 0.09 \\
\hline Yes & $8(12)$ & $2(3)$ & \\
\hline
\end{tabular}


Table 1 Demographic and clinical details, for Indigenous and non-Indigenous patients with cancer of the head and neck in Queensland Australia, 1998- 2004 (Continued)

\begin{tabular}{|c|c|c|c|}
\hline \multicolumn{4}{|c|}{ Hypertension } \\
\hline No & $55(82)$ & $47(76)$ & 0.39 \\
\hline Yes & $12(18)$ & $15(24)$ & \\
\hline \multicolumn{4}{|c|}{ Circulatory disease* } \\
\hline No & $61(91)$ & $51(82)$ & 0.19 \\
\hline Yes & $6(9)$ & $11(18)$ & \\
\hline
\end{tabular}

*Cardiovascular or cerebrovascular disease

medical records, the study is largely descriptive in nature and limited in interpretive power. As a result of matching, Indigenous and non-Indigenous people with head and neck cancer in this study shared a similar demographic profile for location of residence, age and sex, and all attended Queensland public hospitals. We found that only $7 \%$ of Indigenous and $11 \%$ of non-

Table 2 Comparison of cancer treatment given, recurrence, time to death and cause of death, by Indigenous status

\begin{tabular}{|c|c|c|c|}
\hline & $\begin{array}{c}\text { Indigenous } \\
N=67 \\
N(\%)\end{array}$ & $\begin{array}{c}\text { Non- } \\
\text { Indigenous } \\
\mathrm{N}=62 \\
\mathrm{~N}(\%)\end{array}$ & $\begin{array}{c}P \\
\text { value }\end{array}$ \\
\hline \multicolumn{4}{|l|}{ Any Treatment Given } \\
\hline Treatment & $50(75)$ & $59(95)$ & \\
\hline No Treatment & $15(22)$ & $3(5)$ & 0.005 \\
\hline Not sure & $2(3)$ & 0 & \\
\hline Curative treatment given * & $N=59$ & $N=53$ & \\
\hline Curative treatment & $28(48)$ & $40(76)$ & 0.002 \\
\hline No curative treatment & $31(52)$ & $13(24)$ & \\
\hline \multicolumn{4}{|l|}{ Mode of curative treatment $*$} \\
\hline Surgery only & $10(36)$ & $4(10)$ & \\
\hline Chemotherapy only & $1(4)$ & 0 & \\
\hline Radiotherapy only & $5(18)$ & $4(10)$ & 0.03 \\
\hline Surgery and radiotherapy & $7(25)$ & $16(40)$ & \\
\hline $\begin{array}{l}\text { Surgery, chemotherapy and } \\
\text { radiotherapy }\end{array}$ & 0 & $4(10)$ & \\
\hline Chemoradiotherapy & $5(18)$ & $12(30)$ & \\
\hline Recurrence & $N=67$ & $N=62$ & \\
\hline Recurrence recorded & $13(19)$ & $14(23)$ & \\
\hline No recurrence & $49(73)$ & $45(72)$ & 0.77 \\
\hline Not enough information & $5(8)$ & $3(5)$ & \\
\hline \multicolumn{4}{|l|}{ Time from diagnosis to death } \\
\hline Alive at $31^{\text {st }}$ Dec 2006 & $14(21)$ & $30(48)$ & \\
\hline Deceased in $<3$ months & $14(21)$ & $4(7)$ & \\
\hline Deceased 3 month to 12 months & $21(31)$ & $10(16)$ & 0.003 \\
\hline Deceased 12 months to 2 years & $11(17)$ & $8(13)$ & \\
\hline Deceased greater than 2 years & $7(10)$ & $10(16)$ & \\
\hline Cause of death & $N=53$ & $N=32$ & \\
\hline Cancer death & $44(83)$ & $29(91)$ & 0.33 \\
\hline Non cancer death & $9(17)$ & $3(9)$ & \\
\hline
\end{tabular}

*Metastatic cases excluded
Indigenous patients were diagnosed with metastatic disease, in keeping with reports that advanced stage at diagnosis is relatively uncommon for head and neck cancer [14].

Overall, Indigenous people with head and neck cancer were less likely to receive any cancer treatment and when only cases with non-metastatic disease were compared, the treatment disparity was even greater. How this compares with other Indigenous populations with head and neck cancer is not known as no similar published data are available. However, treatment bias among Indigenous people with cancer in general, and with other specific cancers, has been reported in a number of studies [1,15-17]. The reasons why Indigenous people in this study were less likely to receive treatment are not precisely known but we found that those Indigenous people who do not receive treatment were likely to be older, male and socially disadvantaged compared to non-Indigenous people who did not receive treatment. Indigenous people were also less

Table 3 Proportional hazard ratios, using Cox regression models, of time to death for head and neck cancer patients diagnosed between 1998 and 2004, for Indigenous people in Queensland (reference category is last for all variables)

\begin{tabular}{lll}
\hline & HR & $\mathbf{( 9 5 \% ~ C I ) ~}$ \\
\hline Crude Time to all-cause death & $\mathbf{2 . 5 0}$ & $(1.59,3.93)$ \\
Crude Time to cancer-specific death: & $\mathbf{2 . 1 9}$ & $(1.36,3.53)$ \\
$\quad$ Adjusted for: Stage^ & $\mathbf{2 . 4 5}$ & $(1.51,3.96)$ \\
Stage, SEIFA^^ & $\mathbf{2 . 4 1}$ & $(1.47,3.93)$ \\
Stage, SEIFA, CCI score \# & $\mathbf{2 . 3 5}$ & $(1.42,3.78)$ \\
Stage, SEIFA, diabetes & $\mathbf{2 . 3 4}$ & $(1.42,3.85)$ \\
Stage, SEIFA, CCI score, any treatment & $\mathbf{1 . 8 8}$ & $(1.10,3.22)$ \\
Stage, SEIFA, CCl score, curative treatment & $\mathbf{1 . 5 9}$ & $(0.92,2.73)$ \\
Stage, SEIFA, CCl score, mode of treatment\#\# & $\mathbf{1 . 5 0}$ & $(0.86,2.64)$ \\
Crude Time to non-cancer death & $\mathbf{7 . 3 3}$ & $(1.52,35.1)$ \\
Adjusted for: any treatment & $\mathbf{5 . 8 3}$ & $(1.09,31.04)$
\end{tabular}

$\wedge$ Stage: $1=$ localised, $2=$ regional spread, $3=$ distant metastasis, $4=$ not known

$\wedge \wedge$ SEIFA: 1 = Most disadvantaged, $2=$ Mod disadvantage, $3=$ Most Advantaged

\# Charlson Comorbidity Index (CCI) score: $1=0,2=1,3=2-5,4=5+$ \#\# Modes of treatment: $0=$ No Treatment $1=$ surgery, $2=$ chemotherapy only, 3 = radiotherapy only, $4=$ surgery and radiotherapy, $5=$ surgery, chemotherapy and radiotherapy, $6=$ chemoradiation 
likely to receive curative treatment than their non-Indigenous counterparts.

A number of barriers to the use of cancer and other health services for Indigenous Australians have been identified in the literature, and include lack of proximity, availability and cultural appropriateness of health services, lack of specialist care, transport, health insurance and health services affordability, as well as inadequate proficiency in English [16,18-20]. In general these details were not available from the medical records, and are therefore not assessed in this study. Reports suggest factors such as late stage at diagnosis [17], greater prevalence of comorbidities [1] and disparate treatment decisions made by health professionals based on assumptions about socioeconomic and cultural factors [21] may explain some of the treatment differential, but reports are not conclusive.

There was no significant difference in cancer stage or comorbidities between Indigenous and non-Indigenous patients in the present study that might account for the treatment differential, and although we are confident that the medical records were a reliable source of information on cancer stage and the most important comorbidities such as those included in the Charlson index (e.g. diabetes, cardiovascular disease), we acknowledge that our study was small and consequently had limited power to detect small differences between the groups with certainty; consequently some differences may not have been detected. However, it is well known that there is a greater overall burden of comorbidities among the Australian Indigenous population in general and in those with cancer $[1,15]$ and given that Indigenous head and neck cancer patients in this study were 7 times more likely to die from causes other than cancer than non-Indigenous patients, and around 6 times more likely to die from other causes after accounting for any cancer treatment received, it is plausible that chronic ill-health is associated with less treatment.

We acknowledge some possible under-representation of Indigenous patients in this study due to misclassification of Indigenous status at the QCR; however a data quality audit of Queensland hospitals, who provide cancer notification to the QCR, suggest a high level of accurate identification, so this is likely to be minimal [22]. We also believe the Indigenous to non-Indigenous comparison to be internally valid, with little, if any, misclassification of Indigenous status in the study sample, as medical charts were carefully reviewed to verify Indigenous status and discrepant cases were excluded. Unfortunately smoking history was not routinely available from the medical records for this study and we were therefore unable to investigate possible associations between smoking and head and neck cancer for this population. As up to $40 \%$ of Indigenous people are reported to smoke and smoking has been strongly associated with head and neck cancer [23], we recommend more thorough collection of smoking data in the health records of Indigenous patients to enable better assessment of the long-term impact on the health of this population.

Indigenous people who did receive treatment were significantly less likely to receive surgery with adjuvant radiotherapy, the most commonly administered mode of treatment for head and neck cancer in the early stages [4]. However, as mode of treatment varies depending on the tumour site and extent and, as the distribution of cancer types differed somewhat among Indigenous and non-Indigenous people in the present study, it was difficult to compare treatment modes between the two groups. When those who had treatment were compared, there was no difference in dose, duration or completion rate of radiotherapy between Indigenous and non-Indigenous people with head and neck cancer and overall, time to any treatment and curative treatment was also similar. Although numbers were small, the implication is that once engaged in treatment, the treatment pathway was likely to be similar between Indigenous and non-Indigenous people in the public setting in Queensland. This accords with a 2006 report by Condon and colleagues who found that whilst Indigenous patients were less likely to be recommended treatment, there was no significant difference in completion [15].

When cancer-specific death was adjusted for socioeconomic status, the presence and severity of comorbidities and cancer stage, the survival difference remained the same and changed little when adjusted for treatment. However, when curative treatment and mode of treatment were taken into account, the survival difference was no longer significant. This might suggest that if the same treatment was offered, Indigenous people would have similar survival from head and neck cancer as non-Indigenous people. Although the numbers in our cohort were small and so the results lacked statistical precision, we identified disparities in cancer treatment delivery which should be further investigated and addressed by health service personnel. Although survival from head and neck cancer in Indigenous populations has not been reported elsewhere, the results of this study are consistent with the poor cancer survival and worse mortality that has been extensively reported for Indigenous people in Australian with other cancers $[24,25]$. We were unable to adjust for the presence of HPV which has been found to be a predictor of survival in whites in America [26] as HPV testing was not routinely undertaken at the time of the study.

\section{Conclusions}

Despite the small sample size, the study obtained statistically significant and clinically important differences in treatment and survival in Indigenous people with head and neck cancer in Queensland Australia. Indigenous people treated in the public health system in Queensland 
were similar in socioeconomic status and stage of disease to non-Indigenous people and received less treatment and had poorer survival than non-Indigenous people with head and neck cancer. Further study is needed to ascertain to what extent the observed differences may be explained by differences in the true burden of comorbidities in Indigenous and non-Indigenous people.

\section{Abbreviations}

AJCC: American Joint Committee on Cancer; HPV: Human papillomavirus; HR: Hazard ratio; PASW: Statistics Package for the Social Sciences; SCC: Squamous Cell Carcinoma; SEIFA: Socio-economic Indexes for Area; TNM: Tumour, nodes, metastasis; US: United States.

\section{Acknowledgements}

S.M, P.V, G.G and A.G were based at the Queensland Institute of Medical Research. MC was based at the School of Population Health (SPH), University of Queensland (UQ) and the Murdoch Children's Research Institute. SM was supported by a National Health and Medical Research Council (NHMRC) Training Scholarship for Indigenous Australian Health Research (No. 389935), the Perpetual - Derham Green Fund and an NHMRC Project Grant (No. 1004643). PV was supported by an Australian Research Council Future Fellowship (No. 100100511). SM and PV were also supported by the former Australian Centre for International and Tropical Health, UQ. AG was partly supported by a Fellowship from the Medical Research Council (No. 89912). This work was produced as part of the In-Kind activities of the Lowitja Institute incorporating the Cooperative Research Centre for Aboriginal and Torres Strait Islander Health.

\section{Author details \\ 'Cancer and Population Studies, Queensland Institute of Medical Research, Herston Rd, Brisbane, 4060, Australia. ${ }^{2}$ The Australian Centre for International and Tropical Health, University of Queensland, Herston Rd, Brisbane, 4006, Australia. ${ }^{3}$ School of Population Health, University of Queensland, Herston Rd, Brisbane, 4006, Australia. ${ }^{4}$ Murdoch Children's Research Institute, Flemington Rd, Melbourne, 3052, Australia. ${ }^{5}$ Manchester Academic Health Science Centre, University of Manchester, Brunswick St, Manchester, M13 9PL, UK.}

\section{Authors' contributions}

SM made contributions to conception and design, substantial contribution to the acquisition of data, conducted the analysis, interpreted the data and drafted the manuscript. PV made substantial contributions to conception and design, reviewed the analysis and contributed to the interpretation of the data. AG made contributions to conception and design, contributed to the interpretation of the data. GG made contributions to the interpretation of the data. MC made contributions to conception and design, contributed to the interpretation of the data. All were involved in revising it critically for important intellectual content and have given final approval for this version to be published.

\section{Competing interests}

The authors declare that they have no competing interests.

Received: 5 May 2011 Accepted: 25 October 2011

Published: 25 October 2011

\section{References}

1. Valery PC, Coory M, Stirling J, Green AC: Cancer diagnosis, treatment, and survival in Indigenous and non-Indigenous Australians: a matched cohort study. Lancet 2006, 367:1842-1848

2. Cunningham J, Rumbold AR, Zhang X, Condon JR: Incidence, aetiology, and outcomes of cancer in Indigenous peoples in Australia. Lancet Oncology 2008, 9:585-595.

3. Forastiere A, Koch W, Trotti A, Sidransky D: Head and neck cancer. N Engl J Med 2001, 345:1890-1900.

4. Argiris A, Karamouzis MV, Raben D, Ferris RL: Head and neck cancer. Lancet 2008, 371:1695-1709.
5. Australian Institute of Health and Welfare and Australasian Association of Cancer Registries: Cancer in Australia: an overview 2008. Canberra; 2008, Cancer series no. 46 Cat. no. CAN 42.

6. Moore SP, O'Rourke PK, Mallitt KA, Garvey G, Green AC, Coory MD, Valery PC: Cancer incidence and mortality in Indigenous Australians in Queensland, 1997-2006. Med J Aust 2010, 193:590-593.

7. Reichman ME, Kelly JJ, Kosary CL, Coughlin SS, Jim MA, Lanier AP: Incidence of cancers of the oral cavity and pharynx among American Indians and Alaska Natives, 1999-2004. Cancer 2008, 113:1256-1265.

8. Thomas M, Singh J, Scott C, Jayaraj R: Head And Neck Malignancy In The Northern Territory. The Internet Journal of Head and Neck Surgery 2010, 4.

9. Australian Bureau of Statistics: Population Distribution, Aboriginal and Torres Strait Islander Australians 2006. Australian Bureau of Statistics. Darwin; 2007.

10. American Cancer Society: Cancer facts and figures 2002. American Cancer Society. Atlanta; 2002.

11. Charlson ME, Pompei P, Ales KL, MacKenzie CR: A new method of classifying prognostic comorbidity in longitudinal studies: development and validation. Journal of Chronic Diseases 1987, 40:373-383.

12. Australian Bureau of Statistics: Information Paper: Socio-Economic Indexes for Areas (SEIFA) 2001. Canberra; 20032039.

13. de Visscher J, van der Waal I: Etiology of cancer of the lip - A review. International Journal of Oral and Maxillofacial Surgery 1998, 27:199-203.

14. Ferlito A, Shaha AR, Silver CE, Rinaldo A, Mondin V: Incidence and sites of distant metastases from head and neck cancer. Orl; Journal of Oto-RhinoLaryngology \& its Related Specialties 2001, 63:202-207.

15. Condon JR, Cunningham J, Barnes T, Armstrong BK, Selva-Nayagam S: Cancer diagnosis and treatment in the Northern Territory: assessing health service performance for indigenous Australians. Internal Medicine Journal 2006, 36:498-505.

16. Coory M, Green A, Stirling J, Valery P: Survival of Indigenous and nonIndigenous Queenslanders after a diagnosis of lung cancer: a matched cohort study. Medical Journal of Australia 2008, 188:563-566.

17. Hall SE, Bulsara CE, Bulsara MK, Leahy TG, Culbong MR, Hendrie D, Holman CD: Treatment patterns for cancer in Western Australia: does being Indigenous make a difference? Medical Journal of Australia 2004 181:191-194.

18. Condon J: Cancer, health services and Indigenous Australians. Canberra; 2004, 1-26.

19. Prior D: The meaning of cancer for Australian Aboriginal women; changing the focus of cancer nursing. Eur J Oncol Nurs 2009.

20. Newman C, Butow P, Knight R, McCmillan K, Trelour C, Kippax S, Eades S: Cancer and Aboriginal people in Australia: A review of the literature. Critical Public Health 2008, 18:65-75.

21. Boffa JD: Cancer care for Indigenous Australians. Med J Aust 2008, 188:560-561.

22. Australian Institute of Health and Welfare: Indigenous identification in hospital separations data-quality report. Canberra; 2010, Series no. 35. Cat no. HSE 85.

23. Australian Institute of Health and Welfare: National Drug Strategy Household Survey: detailed findings 2004. 2005, no. 16. Cat. no. PHE 66.

24. Condon JR, Barnes T, Armstrong BK, Selva-Nayagam S, Elwood JM: Stage at diagnosis and cancer survival for Indigenous Australians in the Northern Territory. Medical Journal of Australia 2005, 182:277-280.

25. Coory M, Thompson A, Ganguly I: Cancer among people living in rural and remote Indigenous communities in Queensland. Medical Journal of Australia 2000, 173:301-304.

26. Settle $K$, Posner MR, Schumaker $L M$, Tan $M$, Suntharalingam $M$, Goloubeva O, Strome SE, Haddad RI, Patel SS, Cambell EV, et al: Racial survival disparity in head and neck cancer results from low prevalence of human papillomavirus infection in black oropharyngeal cancer patients. Cancer Prev Res (Phila Pa) 2009, 2:776-781.

\section{Pre-publication history}

The pre-publication history for this paper can be accessed here: http://www.biomedcentral.com/1471-2407/11/460/prepub

doi:10.1186/1471-2407-11-460

Cite this article as: Moore et al:: A study of head and neck cancer treatment and survival among indigenous and non-indigenous people in Queensland, Australia, 1998 to 2004. BMC Cancer 2011 11:460. 